Version of Record: https://www.sciencedirect.com/science/article/pii/S1878652919300021

Manuscript_3612b71e780f00396896c88db01fcb27

\title{
La force contraignante de la nomenclature Dintilhac à l'épreuve du principe de réparation intégrale du préjudice
}

\section{Hadrien RACHED ${ }^{1,2}$, Nathalie JOUSSET ${ }^{1,2}$}

${ }^{1}$ Service de médecine légale et médecine pénitentiaire, CHU Angers, 4, rue Larrey, 49933 Angers cedex 09, France

${ }^{2}$ UNAM, université d'Angers, 40, rue de Rennes, BP 73532, 49035 Angers cedex 01, France

Auteur correspondant : Hadrien RACHED hadrien.rached@gmail.com 


\section{Résumé}

La nomenclature Dintilhac, rendue « indispensable » par la loi du 21 décembre 2006 de financement de la sécurité sociale pour 2007, s'est imposée comme l'outil de référence de l'évaluation du dommage corporel permettant une harmonisation et une cohérence en la matière. Depuis peu, la Cour de cassation contrôle le respect de la nomenclature, au visa de l'article 1240 du code civil lui accordant ainsi une force contraignante. Cette prise de position peut générer une violation du principe de réparation intégrale du préjudice, notamment concernant les postes de préjudices intitulés déficit fonctionnel temporaire et déficit fonctionnel permanent. La normativité de la nomenclature Dintilhac, que lui confère le contrôle par la Cour de cassation, sera peut-être définitivement entérinée par la réforme de la responsabilité civile à venir, au risque de sacrifier le principe de réparation intégrale du préjudice.

\section{Abstract}

The Dintilhac nomenclature, made "indispensable" by the Social Security Financing Act of 21 December 2006 for 2007, has established itself as the reference tool for the assessment of bodily injury allowing harmonization and coherence in this area. . Recently, the Court of Cassation checks compliance with the nomenclature, the visa of Article 1240 of the Civil Code thus granting it a binding force. This position may lead to a violation of the principle of full compensation for damage, particularly with regard to the loss items entitled Temporary Functional Deficiency and Permanent Functional Deficiency. The normativity of the Dintilhac nomenclature, conferred on it by the Court of Cassation, will perhaps be definitively confirmed by the reform of the civil liability to come, at the risk of sacrificing the principle of integral compensation for the damage. 


\section{Introduction}

La globalisation de l'assiette de recours des tiers payeurs, qui a prévalu un temps, était sujette à de nombreuses critiques ${ }^{1}$. Les organismes sociaux, dans le cadre de leur action récursoire à l'encontre du responsable du dommage, réclamaient le remboursement des sommes avancées à la victime sur l'indemnité globale due par le responsable. Ainsi, il arrivait que les tiers payeurs (organismes sociaux) récupèrent le montant des sommes avancées sur des postes de préjudices pour lesquels ils n'avaient versé aucune prestation, en particulier sur les préjudices personnels ${ }^{2}$. Ce système était on le comprend défavorable aux victimes.

Dans une logique de protection des victimes d'un dommage corporel, trois lois sont venues effectuer une véritable «révolution copernicienne $»^{3}$ privilégiant les victimes au profit des tiers payeurs.

Premièrement, la loi ${ }^{\circ} 73-1200$ du 27 décembre $1973^{4}$ a exclu les préjudices personnels ${ }^{5}$ du domaine de l'action récursoire des caisses de sécurité sociale, mettant fin au moins partiellement à cette injustice, et amorçant également l'ébauche d'une évaluation des préjudices «poste par poste $»^{6}$.

Deuxièmement, la loi dite Badinter ${ }^{7}$, au terme de ses articles $29^{8}$ et $32^{9}$, a mentionné les organismes pouvant se prévaloir d'une action récursoire, confirmé l'exclusion des préjudices personnels du

${ }^{1}$ Lambert Faivre (Y.), Porchy-Simon (S.), Droit du dommage corporel, Précis, Dalloz, 8ème édition, 2016, 956 p., p. 323 et à suivre.

${ }^{2}$ Prenons l'exemple d'une personne condamnée à verser 100000 euros en dédommagement d'un préjudice corporel. L'organisme social avançait 30000 euros à la victime au titre des frais hospitaliers et des indemnités journalières. Il récupérait ensuite la somme avancée sur les 100000 euros dû par le responsable du dommage, sans se préoccuper de savoir si le montant de la condamnation intégrait 30000 au titre des frais hospitaliers et des indemnités journalières

${ }^{3}$ Porchy-Simon (S.), «1973-1985-2006, les trois temps d'une révolution copernicienne », Gaz, Pal., nº hors-série 1, p. 6.

${ }^{4}$ Loi n ${ }^{\circ} 73-1200 \mathrm{du} 27$ décembre 1973 relative à l'étendue de l'action récursoire des caisses de sécurité sociale en cas d'accident occasionne à un assure social par un tiers, JORF, 30 décembre 1973, p. 14152.

${ }_{5}$ A savoir le préjudice d'agrément, le préjudice sexuel et le pretium doloris.

${ }^{6}$ Moore (J.-G.), «La réparation du préjudice corporel : son évolution de 1930 à nos jours », Gaz. Pal., 2013, n²81.

${ }^{7}$ Loi no ${ }^{85-677 ~ d u ~} 5$ juillet 1985 tendant à l'amélioration de la situation des victimes d'accidents de la circulation et à l'accélération des procédures d'indemnisation, JORF, 06 juillet 1985, p. 7584.

${ }^{8}$ l'article 29 dispose que «Seules les prestations énumérées ci-après versées à la victime d'un dommage résultant des atteintes à sa personne ouvrent droit à un recours contre la personne tenue à réparation ou son assureur: 1. Les prestations versées par les organismes, établissements et services gérant un régime obligatoire de sécurité sociale et par ceux qui sont mentionnés aux articles 1106-9, 1234-8 et 1234-20 du code rural ; 2. Les prestations énumérées au II de l'article ler de l'ordonnance $n^{\circ}$ 59-76 du 7 janvier 1959 relative aux actions en réparation civile de l'État et de certaines autres personnes publiques ; 3 . Les sommes versées en remboursement des frais de traitement médical et de rééducation;4. Les salaires et les accessoires du salaire maintenus par l'employeur pendant la période d'inactivité consécutive à l'événement qui a occasionné le dommage ;5. Les indemnités journalières de maladie et les prestations d'invalidité versées par les groupements mutualistes régis par le code de la mutualité, les institutions de prévoyance régies par le code de la sécurité sociale ou le code rural et les sociétés d'assurance régies par le code des assurances. ».

${ }^{9}$ L'article 32 permet aux employeurs d'exercer une action récursoire sur les responsables du dommage « Les employeurs sont admis à poursuivre directement contre le responsable des dommages ou son assureur le 
domaine cette action, et précisé les indemnités sur lesquelles ils pouvaient exercer une action récursoire. Pour autant, le recours possible se faisait cette fois sur la part de l'indemnité due par le responsable relative aux préjudices patrimoniaux dans leur globalité, alors que pour certains de ces préjudices patrimoniaux, le tiers payeur n'avait versé aucune prestation.

Troisièmement, prenant en compte les rapports Lambert-Faivre de 2003 et Dintilhac de 2005, les observations du médiateur de la République ${ }^{10}$, ainsi que les remarques de la doctrine juridique ${ }^{11}$, le législateur a imposé un « recours poste par poste » aux organismes sociaux par l'article 25, alinéa 1er, de la loi du 21 décembre 2006 de financement de la sécurité sociale pour $2007^{12}$. Sans surprise, les dispositions de cet article soustrayaient en principe les préjudices personnels de l'action récursoire des organismes sociaux ${ }^{13}$, mais admettaient, et c'est là qu'était la nouveauté, que le recours devait se faire sur les préjudices patrimoniaux ayant donné lieu à une véritable prestation indemnitaire, et non plus sur l'ensemble des préjudices patrimoniaux ${ }^{14}$.

On comprend que l'obligation d'un recours poste par poste imposait la création d'une nomenclature, afin d'identifier individuellement chacun des préjudices subis par la victime. Or, une nomenclature de ce type existait déjà au sein rapport Dintilhac. C'est ainsi que la nomenclature éponyme, déjà utilisé par les juridictions, a trouvé toute sa légitimité et son succès ${ }^{15}$.

Cette nomenclature a imposé une triple division des préjudices: selon leur nature directe ou indirecte, patrimoniale ou extrapatrimoniale et, enfin, en fonction de leur temporalité selon la consolidation. Cet outil s'est très rapidement imposé dans le paysage des différentes juridictions civiles $^{16}$, des commissions de conciliation et d'indemnisation $(\mathrm{CCI})^{17}$, des organismes assurantiels ${ }^{18}$

remboursement des charges patronales afférentes aux rémunérations maintenues ou versées à la victime pendant la période d'indisponibilité de celle-ci...».

${ }^{10}$ Proposition de réforme 03-R10 disponible à partir du lien suivant :

http://www.eoi.at/d/EOI\%20\%20Jahresberichte/Frankreich/1115373513_RAPPORT2003[2].pdf

${ }^{11}$ V. Jourdain (P.), «Les tiers payeurs sont admis à exercer un recours sur des indemnités allouées au titre du préjudice fonctionnel que les prestations sociales ne réparent pas », RTD civ., 2004, p.300.

${ }^{12}$ Art. 25, al. 1er, de la loi n ${ }^{\circ} 2006-1640$ du 21 décembre 2006 de financement de la sécurité sociale pour 2007, JORF, $\mathrm{n}^{\circ}$ 296, 22 décembre 2006 p.19315 texte $\mathrm{n}^{\circ} 1$ : «Les recours subrogatoires des caisses contre les tiers s'exercent poste par poste, sur les seules indemnités qui réparent des préjudices qu'elles ont pris en charge, à l'exclusion des préjudices à caractère personnel. »; V. sur le sujet Jourdain (P.), « la réforme des tiers payeurs : des victimes favorisées », D., 2007, p. 454 ; Lienhard (C.), «Recours des tiers payeurs : une avancée législative significative », D., 2007, p. 452.

${ }^{13}$ Sauf exception, voir art. 25, al. 3eme : «Cependant, si le tiers payeur établit qu'il a effectivement et préalablement versé à la victime une prestation indemnisant de manière incontestable un poste de préjudice personnel, son recours peut s'exercer sur ce poste de préjudice.»

${ }^{14}$ Grignon Dumoulin (S.), «L'incidence de l'article 25 de la loi du 21 décembre 2006 sur les recours des organismes sociaux », Droit social, 2008, p. 196.

${ }^{15}$ Bouvet (S.), «Le recours des tiers payeurs », Rev. Fr. Dommage Corp., 2011, n¹, p.69-82.

${ }^{16}$ Circulaire de la DACS n ${ }^{\circ}$ 2007-05 du 22 février 2007 relative à l'amélioration des conditions d'exercice du recours subrogatoire des tiers payeurs en cas d'indemnisation du dommage corporel, p. 5, disponible sur :

http://www.textes.justice.gouv.fr/art_pix/boj_DACS_22\%2002\%2007.pdf.

${ }^{17}$ Délibération du conseil d'administration de l'office le 12 décembre 2007.

${ }^{18}$ Livre blanc sur l'indemnisation du dommage corporel, rédigé par l'Association française de l'assurance, 2008 , disponible sur : http://www.aforcumpsfp.org/doc_utiles/livre_blanc_indem_corporel_avril2008.pdf 
et plus récemment au sein de l'ordre juridictionnel administratif ${ }^{19}$. Le ministère de la justice recommande d'ailleurs son utilisation ${ }^{20}$. Le Fonds d'indemnisation des victimes de l'amiante $(\text { FIVA })^{21}$ et les tribunaux spécialisés de la sécurité sociale restent à ce jour les deux seules entités encore réfractaires à l'utilisation de cet outil ${ }^{22}$.

La reconnaissance quasi unanime et spontanée de cette nomenclature a permis une harmonisation de l'indemnisation des victimes d'un dommage corporel en unifiant et en rationalisant la pratique de son évaluation ${ }^{23}$.

Le succès et les avantages procurés par la nomenclature Dintilhac ne sont pourtant pas dénués d'inconvénients. Ainsi, alors qu'autrefois les juges du fond étaient souverains pour constater l'existence d'un préjudice ${ }^{24}$, la politique jurisprudentielle actuelle de la Cour de cassation consiste en une limitation stricte des préjudices indemnisables aux seuls préjudices mentionnés dans la nomenclature Dintilhac ${ }^{25}$. Les Hauts magistrats brandissent à cet effet le visa de l'article 1382 nouvellement 1240 du code civil ${ }^{26}$, autrement dit le principe de réparation intégrale du préjudice ${ }^{27}$. Cette position de la Cour de cassation est pourtant, paradoxalement, à l'origine d'une violation indirecte de ce principe.

\footnotetext{
${ }^{19}$ CE, 07 octobre 2013, n³37851, Rec. Lebon., RTD civ., 2014, p.131, note P. Jourdain, AJDA, 2014, p. 295, note T. Leleau; CE, 16 décembre 2013, n³46575, Rec. Lebon., AJDA, 2014, p. 524, obs F. Lambolez; RFDA, 2014, p. 317, note C. Lantero.

${ }^{20} \mathrm{http}: / / w w w . j u s t i c e . g o u v . f r / p u b l i c a t i o n s-10047 /$ rapports-thematiques-10049/elaboration-dune-nomenclature-desprejudices-corporels-11945.html

${ }^{21}$ La réparation du dommage corporel répond à un barème propre.

disponible sur: http://www.fiva.fr/documents/Presentation\%20bareme\%20indicatif.pdf. En ce sens Plassard (A.), «Réparation intégrale, FIVA, CAP AT/MP », EN3S, p. 111-118; Pierre (P.), «la nomenclature une dynamique ? Gaz. Pal., 2014, n 361, p.11.

${ }^{22} \mathrm{La}$ réparation est ici forfaitaire : rente ou capital en fonction du taux d'I.P.P. En cas de faute inexcusable de l'employeur avérée la victime peut prétendre à une réparation complémentaire de certains postes de préjudices personnels. En ce sens Moreau (A), «Un (petit) pas vers la réparation intégrale des préjudices consécutifs au AT/MP », Gaz. Pal., 2011, n²2, p. 5 ; Schutze (T.), Bouvet (R.) et Le Gueut (M.A.), «L'évaluation du dommage corporel en cas de reconnaissance de la faute inexcusable de l'employeur », Médecine \& Droit, 2015, n 130, p. 20 à 26 ; Bouvet (R.), Le Gueut (M.A.), «Vers une réparation intégrale de l'accident de travail », Revue de Médecine légale, 2013, n², p. 6570.
}

1. $\quad{ }^{23}$ Gout (O.) et Porchy-Simon (S.), « Plaidoyer pour la défense des nomenclatures dans le droit du dommage corporel », D., 2015, p.1499.

${ }^{24}$ Civ. $2^{\mathrm{e}}, 11$ juillet 1983, n82-12.590 : «attendu que c'est dans l'exercice de son pouvoir souverain d'apprécier les divers chefs de préjudice qu'elle retient et les modalités propres à en assurer la réparation intégrale »; C. Cass., Ass. Plén., 26 mars 1999, n 95-20.640 : «Une cour d'appel apprécie souverainement le montant du préjudice dont elle justifie l'existence par l'évaluation qu'elle en fait, sans être tenue d'en préciser les divers éléments. »; Civ. $1^{\mathrm{re}}, 5$ février 2002, nº 00-12.671, Bull. civ.,: « la cour d'appel [...] a apprécié souverainement le montant du préjudice dont elle a justifié l'existence par l'évaluation qu'elle en a faite, sans être tenue d'en préciser les divers éléments »; sur ce sujet Casson (P.), «Dommages et intérêts », Répertoire de Droit civil, 2017, Chapi. 1, Sect. 1, Art. 1, Para. 1, p. 18-24. ${ }^{25}$ V. Adida-Canac (H.), « le contrôle de la nomenclature Dintilhac par la Cour de cassation », D., 2011, p. 1497.

${ }^{26}$ C. civ., art. 1240 : «Tout fait quelconque de l'homme, qui cause à autrui un dommage, oblige celui par la faute duquel il est arrivé à le réparer. ».

${ }^{27}$ Principe issu de la jurisprudence Civ. $2^{\mathrm{e}}, 28$ octobre 1954, qui s'efforce de le rappeler V. par exemple Civ. $2^{\mathrm{e}}, 23$ janvier 2003, n01-00.200, Bull. civ., JCP, 2003, note Barbiéri. Principe qui a été également mentionné explicitement dans la résolution 75 du Conseil de l'Europe : V. art 1 Conseil de l'Europe Comité des ministres ; Résolution (75) 7 du1 4 avril 1975 relative à la réparation des dommages en cas de lésions corporelles et de décès. 
Après une observation plus détaillée du phénomène susvisé (I), nous esquisserons les solutions envisageables permettant de garantir le respect du principe de réparation intégrale du préjudice (II).

\section{Exposé du problème : une nomenclature normative, contreproductive}

Initialement une simple aide aux juridictions, l'œuvre prétorienne a conféré à la nomenclature Dintilhac un statut normatif.

\section{I.A. La consécration prétorienne de la normativité de la nomenclature}

\section{I.A.1. Une nomenclature facultative, le passé.}

Auparavant, la jurisprudence, bien que s'appuyant sur la nomenclature Dintilhac, n'hésitait pas à s'en écarter si nécessaire. Rappelons-nous ainsi un arrêt de la Cour de cassation rendu en 2006, par lequel elle admettait l'autonomie d'un préjudice d'agrément temporaire ${ }^{28}$. Or, la nomenclature Dintilhac ne retient l'existence d'un préjudice d'agrément qu'après consolidation. Selon la définition de la nomenclature, le préjudice d'agrément temporaire est inclus dans celui de déficit fonctionnel temporaire. Ce dernier regroupant la «perte de qualité de vie et à celle des joies usuelles de la vie courante».

On trouvera un autre exemple de cette «distanciation» vis-à-vis de la nomenclature dans la consécration jurisprudentielle du préjudice moral né du défaut d'information, devenu ensuite le préjudice d'impréparation ${ }^{29}$. Celui-ci n'existe pas dans la nomenclature. Enfin, on retiendra également, toujours selon la Cour de cassation, l'autonomie des différentes déclinaisons du préjudice d'anxiété ${ }^{30}$ et l'autonomie du préjudice d'angoisse de mort imminente, ce qui n'était pas prévu par la nomenclature ${ }^{31}$. La liste est ici bien entendue non exhaustive ${ }^{32}$.

2. ${ }^{28}$ Civ. $2^{\mathrm{e}}, 5$ octobre $2006, \mathrm{n}^{\circ}$ 05-20.139, Bull. civ., RTD civ., 2007, p. 127, obs P. Jourdain.

\footnotetext{
${ }^{29}$ Sur ce préjudice :

En matière civile V. Civ. $1^{\text {re }}, 3$ juin 2010, $\mathrm{n}^{\circ} 09-13.591$, Bull. civ, D., 2010, p.1522, obs. P. Sargos ; RDSS, 2010, p. 898, note F. Arhab-Girardin ; Chroniques de la jurisprudence de la Cour de cassation, 2010, obs. N. Auroy ; D., 2011 , p. 2565, obs. A. Laude; Revue des contrats, 2010, nº 4 , p. 1235 ? note J.S. Borghetti. ; Civ. $1^{\text {re }}, 12$ juillet 2012 , $\mathrm{n}^{\circ} 11-$ 17.510, Bull. civ, D., 2012, p. 2277, obs. M. Bacache ; D., 2013, p. 40, obs. P. Brun et O. Gout ; D., 2012, p. 737, note P. Jourdain. ; Civ. $1^{\text {re }}, 23$ janvier 2014, ${ }^{\circ} 12-22.123$, Bull. civ, RTD civ., 2014, p.379, obs. P. Jourdain ; Lamy de droit civil, 2014, n¹15, note O. Gout ; RDSS, 2014, p.295, note F. Arhab-Girardin ; D., 2014, p. 590, note M. Bacache ; Revue des contrats 2014, ${ }^{\circ}$ 03, p. 168, note A. Guegan-Lecuyer ; LPA, 2014, ${ }^{\circ} 138$, p. 6, note S. Moreil. ; Civ. $1^{\text {re }}$, 15 juin 2016, n 15-11.339, Inédit, Gaz. Pal., 2016, p. 70, note A. Delhaye, RGD, 2017, note S. Hocquet Berg ; Civ. $1^{\text {re }}, 13$ juillet 2016, ${ }^{\circ} 15-19.054$, Revue Lamy Droit civil, ${ }^{\circ} 143$, note J. Amouric. ; Civ. $1^{\text {re }}$, 25 janvier 2017, ${ }^{\circ} 15-27.898$, Bull. civ, RDSS, 2017, p. 716, note D. Cristol; Dalloz actualité, 2017, obs. N. Kilgus; RTD civ., 2017, p.403, obs P. Jourdain ; Gaz. Pal., 2017, n¹6, p.22, obs S. Gerry-Vernières ; Gaz. Pal., n²1, p. 65, obs. A. Delhaye.

En matière administrative V. CE, 10 octobre 2012, 350426, Rec. Lebon, AJDA, 2012, p. 2231, obs. C. Lantero ; RDSS, 2013, p.92, obs. D. Cristol ; LPA, 2013, ${ }^{\circ} 44$, p. 13, note C. Zacharie. ; CE, 16 juin 2016, $\mathrm{n}^{\circ} 382479$, Éditions $^{\circ}$ législatives, 2016, note C. Caillé : «L'impréparation au risque médical survenu : une souffrance morale »; AJDA, 2016, p. 1264, note J-M. Pastor ; Dalloz actualité, 2016, note J-M. Pastor ; D., 2016, p. 1501 note F. Vialla.

${ }^{30}$ V. en autre Soc., 11 mai 2010, n09-42.241, Bull. civ., RTD civ., 2010, p. 534, note P. Jourdain ; D., 2010, p. 2048, note C. Bernard ; D., 2011, p. 35, note P. Brun et O. Gout ; Civ.1ère, 2 juillet 2014 n 10-19.206, Inédit, D., 2014 , p.
} 


\section{I.A.2. Une nomenclature obligatoire, le présent}

Cette souplesse jurisprudentielle semble désormais révolue. Se souciant du respect du principe de réparation intégrale du préjudice - «tout le préjudice mais rien que le préjudice » - la Cour de cassation entérine un changement brutal de trajectoire, en limitant les préjudices indemnisables aux seuls préjudices mentionnés dans la nomenclature Dintilhac. De la sorte, cette nomenclature se voit attribuer une véritable force contraignante ${ }^{33}$. Nombreux sont les exemples qui illustrent cette nouvelle politique. Quatre sont particulièrement édifiant :

- Le refus de l'existence d'un préjudice d'agrément temporaire ${ }^{34}$ et d'un préjudice sexuel temporaire ${ }^{35}$, au motif que ces deux préjudices sont «inclus dans le déficit fonctionnel temporaire ».

- L'inclusion du préjudice d'angoisse des victimes du Distilbene ${ }^{\circledR}$ dans le déficit fonctionnel permanent $^{36}$.

- Le refus par la deuxième chambre de la Cour de cassation ${ }^{37}$ d'autonomiser le préjudice d'angoisse de mort imminente, celui-ci étant considéré inclus dans le pretium doloris et/ou dans le déficit fonctionnel permanent ${ }^{38}$.

2362, note M. Bacache et al ; RCA, 2014, comm. 312, note S. Hocquet-Berg V. sur le sujet Bouvet (R.), Cardinal (M.), Schütze (T.), «Le préjudice d'anxiété né de l'usage d'un produit de santé défectueux », RGDM, n 62, $2017, \mathrm{p} .65-86$. ${ }^{31}$ Crim. 23 octobre 2012, n¹1-83.770, Bull. crim., D., 2014, p. 47, obs P. Brun et O. Gout ; D., 2013, p.1993, obs. J. Pradel, RTD civ., 2013, p. 125, note P. Jourdain ; AJ pénal, 2012, p.657 ; Crim. 15 octobre 2013, n 12-83.055, Bull. crim., Gaz. Pal., 2014, n 56, obs. C. Bernfeld ; Gaz. Pal., n²12, p. 38, obs. A. Mazouz.

${ }^{32}$ On pourra également citer en exemple le préjudice spécifique de contamination jugé distinct du DFP : V. Civ. $2^{\mathrm{e}}, 19$ novembre 2009, n $^{\circ}$ 08-11.622, Bull. civ., RTD civ., 2010, p. 117, note P. Jourdain ; RDSS, 2010, p. 156, note D. Cristol ; RTD com., 2010, p. 601, obs B. Bouloc et Civ. 2e, 18 mars 2010, nº 08-16.169, Bull. civ., RTD com., 2010, note B. Bouloc. Par ailleurs, on pourra objecter que ce préjudice possède une définition jurisprudentielle -«l'ensemble des troubles liés à l'incertitude quant à l'avenir, la crainte de souffrir et les perturbations de la vie intime et sociale » ou «l'ensemble des préjudices de caractère personnel tant physiques que psychiques résultant du seul fait de la contamination virale; qu'il inclut notamment les perturbations et craintes éprouvées, toujours latentes, concernant l'espérance de vie ainsi que la crainte des souffrances; qu'il comprend aussi le risque de toutes les affections opportunistes consécutives à la découverte de la contamination; qu'il comprend également les perturbations de la vie sociale, familiale et sexuelle; qu'il comprend enfin les souffrances, le préjudice esthétique et le préjudice d'agrément provoqués par les soins et traitements subis pour combattre la contamination ou en réduire les effets »- radicalement distincte du préjudice extrapatrimonial évolutif « créé » par la nomenclature Dintilhac, puisque ce dernier possède une composante physiologique, tandis que le second indemnise uniquement souffrance morale inhérente à la connaissance d'un risque de mort en lien avec une pathologie évolutive.

${ }^{33}$ Bacache (M.), «La nomenclature : une norme ? », Gaz. Pal., 2014, n 361, p.7 ; Robineau (M.), « Le statut normatif de la nomenclature Dintilhac des préjudices », JCP G, 2011, n²2, Doctr. 612.

${ }^{34}$ V. Civ. 2e 5 mars 2015, n $14-10.758$, Bull. civ., Gaz. Pal., 2015, p.17, obs A. Guegan-lecuyer ; D., 2015, p. 1791, obs. H. Adida-Canac et al ; D., 2015, p. 2283, obs. M. Bacache - A. Guegan-Lecuyer - S. Porchy-Simon ; D., 2015, p. 35, obs. P. Brun ; Gaz. Pal., 2015, n 181, p. 35, obs. D. Tapinos et Civ. 2e 27 avril 2017, n 16-13.740, Inédit ; Gaz. Pal., 2017, p.63, obs E.Dinparast ; 2016, Rev. Fr. Dommage Corp, p. 95 à 97, note I. Bessieres-Roques.

${ }^{35}$ Civ. $2^{\mathrm{e}}$, 11 décembre 2014, n ${ }^{\circ} 13-28.774$, Bull. civ. ; D., 2015, p. 469, obs. A. Anne Guégan-Lécuyer ; Gaz. Pal., 2015, p. 34, obs. C. Bernfeld ; Gaz. Pal., 2015, n008, p. 24, obs. C. Berlaud.

${ }^{36}$ Civ. $2^{\mathrm{e}}$, 2 juillet 2015, n¹4-19.481, Inédit, Gaz. Pal., 2015, n²53, p. 8, note D. Tapinos; RGD, 2015 , note S. Hocquet Berg.

${ }^{37}$ Civ. $2^{\mathrm{e}}$, 14 septembre 2017, $\mathrm{n}^{\circ}$ 16-22013 ; Civ. $2^{\mathrm{e}}$, 29 juin $2017, \mathrm{n}^{\circ} 16-17.228$, Gaz. Pal., 2017, n³8, p. 68, note E. Dinparast et C. Bernfeld ; Civ. 2e, 2 février 2017, $\mathrm{n}^{\circ}$ 16-11.411, A. Mazouz op. cit.

${ }^{38}$ A noter que la chambre criminelle semble accepter l'autonomie de ce préjudice. Pour une lecture détaillée du sujet voir Porchy-Simon (S.), « L'indemnisation des préjudices situationnels d'angoisse des victimes directes et de leurs proches », 2017, disponible sur : 
- Le refus de l'indemnisation d'un préjudice moral exceptionnel à des fonctionnaires de police victime de violences, la Cour de cassation jugeant que ces souffrances morales étaient inclues dans le déficit fonctionnel permanent ${ }^{39}$.

Le ton est donné, sans jamais la citer explicitement, mais tout en prenant le soin dans ses arrêts de faire sienne les définitions de la nomenclature ${ }^{40}$, la Cour de cassation s'éloigne de sa politique jurisprudentielle antérieure ${ }^{41}$ et juge que tout écart de la nomenclature doit être désormais sanctionné, cassant systématiquement les arrêts d'appel qui prennent des libertés avec celle-ci ${ }^{42}$ au motif de se prémunir de la double indemnisation.

Ce changement de ligne est contestable pour deux raisons. D'une part, il est contraire à l'esprit de la nomenclature. D'autre part, les hauts-magistrats, dans leur volonté d'unifier la pratique du dommage corporel, tombent dans l'écueil d'un traitement mécanique et automatisé des postes de préjudices $^{43}$, qui s'avère nous le verrons en décalage avec l'évaluation médico-légale du dommage corporel. Ce décalage peut être à l'origine d'une mauvaise indemnisation des victimes.

\section{I.B. Les inconvénients de la normativité de la nomenclature}

La décision des magistrats du quai de l'horloge de limiter les postes de préjudices indemnisables à ceux référencés dans la nomenclature Dintilhac suscite des interrogations.

\section{I.B.1. Une normativité contraire à la volonté des rédacteurs}

Il convient de rappeler tout d'abord que le groupe de travail à l'origine de cette nomenclature ne souhaitait pas qu'elle devienne un «carcan rigide et intangible conduisant à exclure systématiquement tout nouveau chef de préjudice sollicité dans l'avenir par les victimes » mais, au

http://www.ajdommagecorporel.fr/sites/www.ajdommagecorporel.fr/files/fichier_cv/Rapport\%20sur\%201\%27indemnis ation $\% 20 \mathrm{des} \% 20 \mathrm{pr} \% \mathrm{C} 3 \%$ A 9 judices $\% 20$ situationnels $\% 20 \mathrm{~d} \% 27$ angoisse $\% 20 \mathrm{des} \% 20 \mathrm{victimes} \% 20 \mathrm{directes} \% 20 \mathrm{et} \% 20 \mathrm{de}$ $\% 201$ urs $\% 20$ proches.pdf

3. $\quad{ }^{39}$ Civ. $2^{\mathrm{e}}, 5$ mars $2015, \mathrm{n}^{\circ} 14-10.758$, Bull. civ., D., 2015, p. 1791, obs. H. Adida-Canac et al ; D., 2015, p. 2283, obs M. Bacache - A. Guegan-Lecuyer - S. Porchy-Simon ; D., 2016, p.35, obs. P. Brun- O. Gout ; Gaz. Pal., 2015, n99, p. 5, note A. Guegan-Lecuyer; Gaz. Pal., 2015, n 106, p.20, note S. Gerry-Vernières.

${ }^{40}$ Alvarez (L.), Arlabosse (R.), Ferro (R.), «Le recours des tiers payeurs depuis la loi du 21 décembre 2006 : une évolution en demi-teinte », 2011, disponible sur :

http://www.alvarez-arlabosse.com/wp-content/uploads/2015/09/Recours_des_tiers_payeurs.pdf

${ }^{41}$ En ce sens : Crim., 3 novembre 1955, D., note R. Savatier ou Crim., 4 février 1970, n68-93.464, Bull. Crim. «attendu que, si, en matière de dommages provenant d'un crime ou d'un délit, les juges de répression apprécient souverainement, dans les limites des conclusions de la partie civile, l'indemnité due à celle-ci, ils ne sauraient se référer, dans une espèce déterminée à des règles établies à l'avance pour justifier leur décision »; plus proche de nous Civ. 2 ${ }^{\mathrm{e}}$, 22 novembre 2012, n ${ }^{\circ} 11-25.988$, Bull. civ., Gaz. Pal., 2013, obs. C. Bernfeld.

${ }^{42}$ D'autres exemples de l'inflexibilité des magistrats du quai de l'horloge : Civ. $2^{\mathrm{e}}$, 18 mai 2017, n 16-11.190, Bull. civ., Gaz. Pal., 2017, n 38, p. 64, note C. Bernfeld ; Civ. 2e 5 mars 2015 14-13.045, Inédit, Gaz. Pal., 2015, n 181, p. 34, note M. Perini Minsky.

${ }^{43}$ Arcadio (D.), « Abaques, tables, barèmes et autres référentiels d'indemnisation... », Gaz. Pal., 2011, n³37, p. 10. 
contraire, qu'elle s'appréhende plutôt comme «- une sorte de guide - susceptible au besoin de $s^{\prime}$ enrichir de nouveaux postes de préjudices qui viendraient alors s'agréger à la trame initiale ${ }^{44}$. Elle devait être purement indicative et non limitative afin de ne pas contraindre les acteurs du dommage corporel $^{45}$. Le préjudice permanent exceptionnel que la nomenclature laissait explicitement à l'appréciation souveraine des juges et des médecins chargés d'évaluer le dommage est le témoin de la volonté de souplesse et d'évolutivité voulue par le groupe de travail ${ }^{46}$.

En rendant impossible la création de nouveaux postes de préjudices, ou en empêchant l'autonomisation de ceux prévus par la nomenclature, il apparait donc que les magistrats aient «dissous l'esprit de la nomenclature pour le besoin d'une sécurité juridique »"7.

\section{I.B.2. Une normativité au détriment des victimes}

Toutefois, de prime abord, la position de la Cour de cassation pourrait sembler pertinente. Reprenons les exemples précités. Eu égard à la largesse de sa définition dans la nomenclature, le déficit fonctionnel temporaire - perte de qualité de vie et à celle des joies usuelles les de la vie courante $^{48}$ - inclut bien la notion de préjudice d'agrément temporaire ainsi que la notion de préjudice sexuel temporaire. De même, le déficit fonctionnel permanent - les atteintes aux fonctions physiologiques de la victime [...] la douleur permanente qu'elle ressent, la perte de la qualité de vie et les troubles dans les conditions d'existence qu'elle rencontre au quotidien après sa consolidation $^{49}$ - subsume la notion de souffrance morale.

On pourra objecter, néanmoins, que la réalité de l'évaluation du dommage corporel qui est faite par les médecins experts est en décalage avec les catégories du rapport Dintilhac. En effet, comme nous l'avons dit précédemment, le déficit fonctionnel temporaire, comme le déficit fonctionnel permanent, ont la particularité de présenter à la fois « un aspect fonctionnel objectif » et un « aspect situationnel, au contraire très subjectif $»^{50}$. Le fonctionnel, c'est le corps ; le situationnel, c'est le vécu du dommage. Or, la classification du déficit fonctionnel temporaire proposée par l'Association

\footnotetext{
${ }^{44}$ Rapport Dintilhac, p. 4, disponible sur : http://www.ladocumentationfrancaise.fr/var/storage/rapports-publics/064000217.pdf

${ }^{45}$ Rapport Dintilhac, p. 12 ; En ce sens également Rapport Lambert-Faivre, page 12 dont le rapport Dintilhac c'est inspiré disponible sur : http://www.ladocumentationfrancaise.fr/var/storage/rapports-publics/034000490.pdf

${ }^{46}$ Tapinos (D.), « Le préjudice d'agrément temporaire en quête d’autonomie », Gaz. Pal., 2015, n¹27, p. 10

4. $\quad{ }^{47}$ Cousin (C.), « Les nomenclatures des préjudices corporels : comment ressusciter l'esprit du rapport Dintilhac ? Propositions pour une évolution de la nomenclature des postes de préjudices à partir d'un arrêt de la première chambre civile de la Cour de cassation du 15 juin 2016 », Revue Lamy Droit civil, n 147, 2017, p.

${ }^{48}$ Rapport Dintilhac, p. 38.
${ }^{49}$ Rapport Dintilhac, p. 38.
}

5. $\quad{ }^{50}$ Guégan-Lécuyer (A.), « Le préjudice sexuel aux prises avec le déficit fonctionnel temporaire », D., 2015, p. 469. 
pour l'étude de la réparation du dommage corporel (AREDOC) ${ }^{51}$, ainsi que les barèmes d'évaluation de l'invalidité (et donc du déficit fonctionnel permanent) n'intègrent pas l'aspect situationnel $^{52}$. Ces outils, créés pour la plupart bien avant la nomenclature Dintilhac ${ }^{53}$, ne donnent «qu'une mesure objective basée sur un étalonnage physiologico-fonctionnel du corps humain » ${ }^{54}$ sans prendre en compte le regard plus psychologisé s5 $^{5}$, plus subjectivisé ${ }^{56}$ de notre société sur le dommage corporel, la composante physiologique n'étant désormais qu'une facette parmi d'autre du dommage corporel.

Si les experts chargés d'évaluer le dommage peuvent se détacher de la lecture exclusivement physiologique proposée par ces outils ${ }^{57}$, cette «équation audacieuse », ou de manière plus cynique cette «addition de pommes et de poires $»^{58}$, qui réside dans l'amalgame de plusieurs éléments de différentes natures au sein des déficits fonctionnels temporaire et permanent ${ }^{59}$ - éléments objectifs/physiologiques et éléments subjectifs/relatifs au vécu du dommage - complique leur travail. Le soin de majorer ou non le taux d'I.P.P. proposé par les barèmes pour le DFP, ou la classe de l'AREDOC pour le DFT, en y incluant pour le premier les «souffrances endurées » post consolidation et la perte de qualité de vie et pour le second le préjudice d'agrément temporaire et le préjudice sexuel temporaire, est laissé à leur totale discrétion ${ }^{60}$.

Cet amalgame peut conduire, en conséquence, à une minimisation de certaines composantes du DFT et du DFP, plus particulièrement concernant l'aspect situationnel, eu égard à la difficulté intrinsèque de son évaluation ${ }^{61}$ et à la déficience des outils d'évaluation proposés.

\footnotetext{
${ }^{51}$ Lettre de l'AREDOC, disponible sur :

http://www.aredoc.com/wp-content/uploads/2017/08/Point-12-PDF.pdf
}
6. $\quad{ }^{52}$ Van Teslaar (S.), «L'indemnisation imparfaite du déficit fonctionnel permanent », D., 2017, p.1309 ; Porchy- Simon (S.), «Quelles amélioration pour la nomenclature Dintilhac ? Le point de vue de l'universitaire... », Gaz. Pal., 2011, p.19 ; Lopez (G.), « les barèmes d'évaluation sont-ils obsolètes ? », Gaz. Pal., 2015, n48, p. 48

\footnotetext{
531980 pour le barème du concours médical révisé en 2001 et 1991 pour le barème de la Société Française de médecine Légale révisé en 2000 / Nomenclature Dintilhac 2005.

${ }^{54}$ Rapport Lambert-Faivre, page 10, disponible sur :

http://www.ladocumentationfrancaise.fr/var/storage/rapports-publics/034000490.pdf

${ }^{55}$ Molfessis (N.), «La psychologisation du dommage », In Quel Avenir pour la responsabilité civile ?,Thèmes et commentaires, Dalloz, 2015, p. 39 et suivantes, 146 p.

${ }^{56}$ Bouvet (R.), Cardinal (M.), Schütze (T.), «Le préjudice d'anxiété né de l'usage d'un produit de santé défectueux », $R G D M, \mathrm{n}^{\circ} 62,2017$, p. 65-86

${ }^{57} \mathrm{Si}$ tant est qu'ils aient connaissance de cette inadéquation.

${ }^{58}$ Arcadio (D.), «Les bons ouvriers ont toujours de bons outils (ou comment améliorer les outils de la réparation du dommage corporel), colloque La réparation du dommage corporel à l'épreuve de l'unification des pratiques », Gaz. Pal., 2011, n³58, p.24.

${ }^{59}$ Jourdain (P.), « Conclusion prospective », Gaz. Pal., 2014, n³61, p. 36.
}

1. $\quad{ }^{60}$ Coviaux (A.), « La nomenclature Dintilhac, la belle aubaine ! », AJ pénal, 2017, p. 8 qui insiste sur le fait de vérifier la prise en compte de ses éléments situationnels.

${ }^{61}$ Prévost (J.B.), « La difficile mesure de la qualité de vie », Gaz. Pal., 2011, nº197, p.22. 
On observe ainsi un véritable décalage entre la réalité médico-légale de l'évaluation de ce préjudice et la définition proposée par la nomenclature telle que retenue par les magistrats.

Finalement, les magistrats n'avaient pas voulu indemniser certains préjudices (agrément temporaire, sexuel temporaire...) afin de ne pas violer le principe de réparation intégrale du préjudice en enrichissant indument le requérant. Cependant, du fait l'existence du décalage observé, ceux-ci peuvent finalement transiger avec le principe de réparation intégrale du préjudice, en appauvrissant la victime si ces éléments n'ont pas été pris en compte dans le calcul du taux d'I.P.P. ou dans les classes dégressives de l'AREDOC.

En tout état de cause, "l'hétérogénéité ${ }^{62} \mathrm{du}$ déficit fonctionnel temporaire et du déficit fonctionnel permanent corrélée aux outils fournis aux praticiens chargés d'évaluer le dommage, sont de nature à limiter l'évaluation de ces préjudices et donc l'indemnisation qui en découle.

C'est pourquoi la nomenclature qui avait été proposée afin de respecter au mieux le principe de réparation intégrale du préjudice est devenue, de manière paradoxale, en raison de la politique jurisprudentielle actuelle, l'artisan possible de sa transgression.

\section{Une problématique, de nombreuses solutions}

Cette situation n'est pas inéluctable. Il existe des solutions envisageables pour venir à bout des imperfections de la nomenclature et/ou des outils d'évaluation du dommage corporel. Certaines sont conformes à l'esprit de la nomenclature Dintilhac (II.A), tandis que d'autres nécessitent une rupture avec celle-ci (II.B).

\section{II.A. Les solutions gardant l'esprit de la nomenclature}

Deux solutions, qui ne transigeront pas avec l'esprit de la nomenclature, sont envisageables pour venir à bout de l'écueil qui réside dans la globalisation des préjudices au sein des déficits fonctionnels temporaire et permanent.

\section{II.A.1. La voie de l'optimisation des outils d'évaluation du dommage}

On pourrait apporter de nouveaux outils qui viendraient suppléer les lacunes de la classification du DFT proposée par l'AREDOC et des barèmes d'évaluation de l'invalidité, afin que l'aspect situationnel de ces préjudices soit pris en compte. Le but est de permettre une véritable adéquation entre la jurisprudence et les définitions proposées pour ces préjudices par la nomenclature, d'une part, et la réalité de l'évaluation médico-légale du dommage corporel, d'autre part.

En ce sens, Daphné Tapinos ${ }^{63}$ rappelle que certains auteurs ont proposé une approche plus descriptive de ces préjudices ${ }^{64}$ afin d'appréhender toute leur composante subjective, en

\footnotetext{
${ }^{62}$ Porchy-Simon (S.), « l'articulation des postes de préjudice », Gaz. Pal., 2014, n³61, p. 24

${ }^{63}$ Tapinos (D.), op. cit.

${ }^{64}$ Approche préconisée depuis longtemps Barrot (R.), « Le dommage corporel et sa compensation », Litec, 1998.
} 
«fiabilisant» cette description par l'utilisation de questionnaires complet intégrant toutes les dimensions des déficits fonctionnels ${ }^{65}$, ou par l'emploi de logiciels comme Handistest ${ }^{66}$. Tous s'accordent et plaident, en complément de l'optimisation des outils d'évaluation, pour une collégialité de l'expertise, associant médecins et d'autres acteurs de la santé, afin d'apprécier le plus finement ces préjudices, à l'instar par exemple de l'évaluation des besoins des personnes handicapées qui a lieu au sein des équipes pluridisciplinaires des $\mathrm{MDPH}^{67}$.

Cette solution s'articulerait efficacement avec la «mission d'expertise, au terme de laquelle l'expert devrait évaluer [les composantes du DFT et du DFP] qui paraissent de nature différente » proposée par Claudine Bernfeld et Fréderic Bibal ${ }^{68}$.

Ainsi, l'expert à l'aide des nouveaux outils mis à sa disposition pourrait donner :

- Pour le DFT:

- une évaluation en pourcentage de la gêne temporaire partielle en utilisant la classification actuelle proposée par l'AREDOC,

- $\quad$ une description et un chiffrage sur une échelle de 0 à 7 du préjudice sexuel temporaire ${ }^{69}$,

- une description de la perte de la qualité de vie (et donc par conséquent une description d'un éventuel préjudice d'agrément temporaire), à l'aune des questionnaires en question.

- $\quad$ Pour le DFP :

- une évaluation en pourcentage des séquelles à l'aide des barèmes actuels,

- $\quad$ un chiffrage des souffrances endurées sur une échelle de 0 à 7 ,

- $\quad$ une description de la perte de qualité de vie à la lumière des nouveaux questionnaires.

\section{II.A.2. Une solution prétorienne}

Une autre solution passe par la voie prétorienne. La jurisprudence peut défaire ce qu'elle a fait ${ }^{70}$. Tout d'abord, et c'est le point le plus important, les magistrats pourraient effectuer un revirement de jurisprudence en conférant à la nomenclature Dintilhac un caractère simplement indicatif en la

\footnotetext{
${ }^{65}$ Gras (M.P.), Guillon (B.), « Proposition pour une indemnisation concrète du DFT », Gaz. Pal., 2011, n 197, p.16

${ }^{66}$ Hamonet (C.), «Pour une nouvelle méthodologie et une humanisation de l'expertise médicale », Gaz. Pal., 2008 $\mathrm{n}^{\circ} 110$, p.62

${ }^{67}$ Maison Départementale des personnes handicapées créée par la loi n ${ }^{\circ}$ 2005-102 du 11 février 2005 pour l'égalité des droits et des chances, la participation et la citoyenneté des personnes handicapée, JORF, n³6, 12 février 2005, p.2353, texte $\mathrm{n}^{\circ} 1$.

${ }^{68}$ Bernfeld (C.), Bibal (F.), « Présentation : le déficit fonctionnel permanent, une trinité », Gaz. Pal., 2011, n 357, p. 6. ${ }^{69}$ Avec un certain risque relatif de barémisation qui réside dans l'association du chiffrage sur une échelle de 0 à 7 et dans les référentiels d'indemnisation; V. F. Bibal, «Fiches pratiques XII : souffrances endurées », Gaz. Pal., n³1, p. 41; référentiel Mornet disponible sur :http://www.ajdommagecorporel.fr/sites/www.ajdommagecorporel.fr/files/fichier_cv/Referentiel-Mornet-2015.pdf ${ }^{70}$ Arcadio (D.), «Préjudice d'agrément, préjudice sexuel et autres menus déplaisirs... Réflexions à propos des préjudices extrapatrimoniaux dans la nomenclature DINTILHAC » disponible sur : http://www.arcadioavocats.fr/piecesjointes-contenu/prejudices_extrapatrimoniaux.pdf
} 
citant explicitement. Le but est de garantir une caractérisation des préjudices intuitu personae par les juges du fond, en ouvrant la porte à un enrichissement de la nomenclature ${ }^{71}$ par de nouveaux postes de préjudices au cas par cas.

Ensuite, les magistrats pourraient effectuer un travail de refonte de la nomenclature. Ce travail est nécessaire afin de permettre l'autonomisation de certains postes de préjudice du DFT et du DFP, qui serait bénéfique à double titres.

D'une part, cela permettrait de mettre fin, au moins en partie, à la dysmétrie entre les postes de préjudice avant consolidation et après consolidation.

Cela irait dans le sens d'une «continuité temporelle ${ }^{72}$ et d'une simplification salutaire de la nomenclature ${ }^{73}$. N'est-il pas étonnant que le préjudice esthétique jouisse d'une autonomie aussi bien avant qu'après consolidation, tandis que le préjudice sexuel ou d'agrément ne s'appréhende qu'après consolidation, ou que les souffrances endurées ne soient autonomes qu'avant consolidation $^{74}$ ? Comme le souligne Marie Badel $^{75}$, les définitions très strictes données à ces postes de préjudices par la nomenclature, ainsi que par la jurisprudence, ne laissent aucun doute sur leur autonomie. Leur inclusion avant consolidation dans le DFT, et leur inclusion après consolidation dans le DFP, sont bien ici le signe d'une incohérence.

D'autre part, l'autonomisation de certains aspects du DFT et du DFP permettrait une évaluation bien plus fine de ces derniers par les médecins experts que sous l'actuel amalgame au sein du DFT et DFP. Ces médecins seraient en effet «contraints » de les évaluer, alors que sous l'amalgame actuel cela n'est aucunement garanti et n'est d'ailleurs pas vérifié.

Le déficit fonctionnel «a minima $»^{76}$, c'est-à-dire sans son aspect situationnel, serait dès lors finement appréhendé avec les outils d'évaluation du dommage actuels mis à la disposition des experts du dommage corporel (barèmes d'indemnisation du dommage corporel/classification de l'AREDOC).

\footnotetext{
${ }^{71}$ V. Philippe Brun « s'il y a tout lieu de prendre très au sérieux cette nomenclature, comme l'ont déjà fait la jurisprudence, la doctrine et la pratique, il faut aussi se garder d'un excès de révérence pour cet instrument qui ne marque en rien une fin de l'histoire. La qualité de l'indemnisation est un combat de tous les jours. La nomenclature doit être conçue, il me semble, comme un laboratoire, un chantier permanent », Gaz. Pal., 2014, n'361, p. 21.
}

2. $\quad{ }^{72}$ Mireille Bacache (M.), Anne Guégan-Lécuyer (A.) et Porchy-Simon (S.), « Dommage corporel », D., 2015, p. 2283.

${ }^{73}$ Porchy-Simon (S.), « Plaidoyer pour une construction rationnelle du droit du dommage corporel », D., 2011 , p. 2742.

${ }^{74}$ En ce sens Viney (G.), Jourdain (P.), Carval (S.), «Les effets de la responsabilité », LGDJ, « Traités », 2017,880 p.

3. $\quad{ }^{75}$ Badel (M.), « Faute inexcusable * Indemnisation complémentaire * Souffrances physiques et morales * Préjudice d'agrément », RDSS, 2013, p. 359.

${ }^{76}$ Guegan-Lecuyer (A.), «La distinction de préjudices temporaires et permanents : l'exemple du déficit fonctionnel », Gaz. Pal., 2014, n 361, p. 28. 
Certains verront dans cette proposition une énième «poussée du besoin indemnitaire ${ }^{77}$ caractérisée par l'inflation des préjudices indemnisables ${ }^{78}$. S'il est certain que «diviser un préjudice ne crée pas pour autant un droit supplémentaire ${ }^{79}$, ce que certains prénomment l'atomisation du dommage $^{80}$ ou encore la dérive nominaliste ${ }^{81}$ est peut-être dans notre présent cas un maigre sacrifice pour conserver une cohérence dans la réparation du dommage corporel et, surtout, afin de garantir un respect plus strict du principe de réparation intégrale du préjudice. S'il est vrai que ce principe n'a pas de valeur constitutionnelle ${ }^{82}$, et qu'il parait certainement artificiel de vouloir indemniser intégralement l'atteinte à la vie humaine ${ }^{83}$ : en effet si ce principe s'accommode particulièrement bien des préjudices patrimoniaux puisqu'il suffit alors de s'adonner à des calculs mathématiques, son application repose en matière personnelle sur une théorie du magistrat, une théorie selon laquelle le montant qu'il alloue pour les préjudices personnels les répare dans leur entièreté ${ }^{84}$. Bien qu'un simple dogme - admise à demi-mot par la Cour de cassation qui reconnait son indemnisation comme «de principe ${ }^{85}$, l'application du principe de réparation intégrale du préjudice, même en matière personnelle, demeure une «utopie constructive ${ }^{86}$, autrement dit un symbole ${ }^{87}$ qui va au-delà de la simple indemnisation pécuniaire, aspirant surtout à la reconnaissance de l'identité, de l'individualité de la victime ${ }^{88}$, en d'autres termes à sa consolation ${ }^{89}$.

\footnotetext{
${ }^{77}$ Le Tourneau (P.), « Responsabilité (en général) », Rép. Civ. Dalloz, 2001

78 Cadiet (L.), «Sur les faits et les méfaits de l'idéologie de la réparation », D., 2000, p. 495-510 ; Mazeau (D.), « Réflexions sur un malentendu », D., 2001, p. 332.
}

${ }^{79}$ Chifflet (B.), « Le point de vue du magistrat judiciaire sur la mise en place d'une nomenclature unique des postes de préjudices réparables, colloque La réparation du dommage corporel à l'épreuve de l'unification des pratiques », Gaz. Pal., n³58, p. 27.

${ }^{80}$ Le Tourneau (P.), « La responsabilité civile : «Que sais-je ?»» n 1521. Presses universitaires de France, 2003.

${ }^{81}$ Fabre-Magnan (M.), « le dommage existentiel », D., 2010, p. 2376.

${ }^{82}$ Cons. const., 22 octobre 1982, n82-144-QPC, note D. Le Prado ; Cons. const., 20 juillet 1983, n 83-162 ; Cons. const., 18 juin 2010, n² 2010-8-QPC ; Constitution, 2010, p. 413, note C. Radé ; D., 2011, p. 459, note S. PorchySimon.

${ }^{83}$ Résumé par la phrase d'André Malraux dans les conquérants : « la vie ne vaut rien, rien ne vaut la vie ». V. également Viney (G.), «Quelques propositions de réforme du droit de la responsabilité civile » D., 2009, p. 2944 ; Leduc (F.), Pierre (P.), La réparation intégrale en Europe: Études comparatives des droits nationaux », Larcier, ELSB EUROPE(S), 2011 : «prétendre en d'autres terme que la réparation doit être strictement équivalente aux souffrances physiques et morales endurées par la victime, relève plus de la théorie des actes de langage que de la réalité : la réparation n'est intégrale que parce que le juge a dit qu'elle l'était, mais, en réalité, son montant est nécessairement fixé de façon totalement arbitraire »; V. également G. Viney et P. Jourdain, Les effets de la responsabilité, 4eme éd., LGDJ, 2017, p.116 et s. et p. 355.

${ }^{84}$ Groutel (H.), « Réparation intégrale et barémisation : l'éternelle dispute », $R C A, 2006, \mathrm{n}^{\circ} 11$, repère 11.

${ }^{85}$ Civ. $2^{\mathrm{e}}, 28$ novembre 1962, $\mathrm{n}^{\circ} 756$, Bull. civ., «...mais que la douleur morale se prêtait mal à une réparation pécuniaire qui, pour être juste ne pouvait être que de principe... ».

${ }^{86}$ Propos rapporté à Jean Pierre Dintilhac.

${ }^{87}$ Philippe Brun compare le principe de réparation intégrale du préjudice à la force obligatoire de l'article 1314 du code civil dans le droit des contrats V. Brun (P.), «Responsabilité du fait personnel », 2015

${ }^{88}$ Denimal (M.), «La réparation intégrale du préjudice corporel : réalités et perspectives ». Droit. Université du Droit et de la Sante - Lille II, 2016, p. 23.; Lambert-Faivre (Y.), Porchy-Simon (S.), Droit du dommage corporel, Précis, Dalloz, 8ème édition, 2016, p. 24.

${ }^{89}$ Corgas-Bernard (C.), « Le préjudice d'angoisse consécutif à un dommage corporel : quel avenir ?», $R C A, 2010, \mathrm{n}^{\circ} 4$, étude 4 citant Carbonnier (J.), Droit civil, t. 4, Les obligations : PUF, n 208, p. 384 ; 
Si les pistes que nous venons de développer sont susceptibles de corriger la problématique soulevée, cette correction pourrait également passer par une rupture totale avec la nomenclature Dintilhac.

\section{II.B. La globalisation des préjudices ou l'édiction d'une nouvelle nomenclature}

\section{II.B.1. Le retour à la globalisation des préjudices}

Certains auteurs, inquiets de la multiplication des postes de préjudice, suggèrent d' «appréhender les répercussions strictement personnelles du dommage corporel au moyen d'un chef de préjudice unique ${ }^{90}$. Il n'existerait alors qu'un unique préjudice personnel global, à déterminer de manière individuelle pour chaque victime ${ }^{91}$. Il s'agit ici non pas d'une addition des différents chefs de préjudices composant ce préjudice unique mais bien en une appréciation globale de ce dernier. Cette solution est notamment celle du droit québécois ${ }^{92}$, du droit allemand ${ }^{93}$ ou encore du droit Anglais ${ }^{94}$.

Cette solution présente plusieurs avantages. En premier lieu, le choix d'appréhender les préjudices personnels en un unique préjudice global permet d'intégrer les nouvelles formes d'atteintes personnelles, sans qu'il soit nécessaire de créer à chaque fois un nouveau poste de préjudice spécifique pour les indemniser, puisqu'il suffit alors d'augmenter le quantum du préjudice personnel global pour intégrer son indemnisation ${ }^{95}$. En deuxième lieu, comme l'a démontré le

\footnotetext{
${ }^{90} \mathrm{~V}$. Knetsch (J.), « La désintégration du préjudice moral », D., 2015, p. 443 ; Le Tourneau (P), « Droit de la responsabilité et des contrats - Régimes d'indemnisation », Dalloz, « Dalloz action » 2017, 11eme édition, 2702 p.

${ }^{91}$ Gout (O.), «La mise en place d'une nomenclature unique des postes de préjudices repérables », Gaz. Pal., 2011, $\mathrm{n}^{\circ} 358$, p. 9.

${ }^{92}$ Gardner (D.), «Le préjudice extrapatrimonial : convergences et divergences des droits québécois et français », Gaz.

Pal., 2016, n³2, p. 58 ; Arcadio (D.), « Regards croisés sur l'appréciation des préjudices extrapatrimoniaux au Québec

et en France », Gaz. Pal., 2016, n³2, p. 64.

${ }^{93}$ En Allemagne en effet, l'ensemble des préjudices extrapatrimoniaux sont indemnisés sous la forme d'un poste unique le Schmerzensgeld que l'on peut traduire par «l'argent de la douleur »; V. Schutte (M.), « 1ère partie : L'influence de la résolution du Conseil de l'Europe de 1975 sur la pratique européenne L'expérience de l'avocat allemand », Gaz. Pal., 2010, n¹00, p.10.

${ }^{94} \mathrm{Ce}$ préjudice extrapatrimonial unique est appelé non-pecuniairy losses/pain and suffering loss of amenity. Il est indemnisé de manière forfaitaire et plafonné (avec donc l'admission du caractère non compensable du préjudice extrapatrimonial), une seule atteinte prouvée d'une ses composantes donne accès à l'indemnisation V. Rossetti (M.), «L'indemnisation du préjudice corporel et l'équité : les "racines communes" des règlementations européenne », p. , disponible sur : https://www.courdecassation.fr/IMG/File/pdf_2006/05-122006_assurance/05-12-06_marco_rossetti.pdf

${ }^{95}$ Gardner (D.), op. cit. ; Prévost (J.B.), « Réflexions venues d'ailleurs : comparaison entre les droits du dommage corporel québécois et français», Gaz. Pal., 2016, n032, p. 75.
} 
Professeur Daniel Gardner, la globalisation des préjudices extrapatrimoniaux peut conduire à une indemnisation supérieure à la nôtre ${ }^{96}$.

Cependant, en l'absence d'outils idoines mis à disposition des médecins experts afin d'évaluer toutes les facettes des troubles dans les conditions d'existence, autrement dit la gêne occasionnée dans la vie courante de la victime ${ }^{97}$, cette solution remettrait en cause du principe de réparation intégrale du préjudice. La globalisation de l'ensemble des répercussions personnelles, moulées dans un préjudice unique, ne ferait qu'aggraver la situation que nous avons soulevée plus haut. Le médecin expert ne pourrait pas évaluer finement cet amalgame et éclairer comme il se doit le juge du fond en charge de l'affaire. On a vu que les outils actuels ne le permettent pas, puisqu'ils n'intègrent pas les aspects situationnels liés au dommage. Sans un outil adéquat, le médecin ne peut évaluer correctement le préjudice unique intégrant les souffrances physiques et morales du patient, le retentissement fonctionnel de son dommage, sa perte de la qualité de vie, son retentissement ou non sur l'activité sexuelle, etc.

Par ailleurs, l'indemnisation unique d'un amalgame de préjudices différents empêche de connaitre le raisonnement des magistrats pour parvenir au montant de cette indemnisation ${ }^{98}$. Comment alors contrôler que les magistrats ont bien respecté le principe de réparation intégrale du préjudice ? Comment alors connaître les facteurs ayant motivé la décision des magistrats ? Cette méthode dite de la globalisation des préjudices serait, en l'état actuel des outils d'évaluation du dommage corporel, un retour en arrière, sur le plan de l'indemnisation, et aussi sur le plan de la «transparence » judiciaire. C'est pourquoi, à l'heure actuelle, la ventilation des préjudices par le biais d'une nomenclature apparait comme le meilleur moyen de permettre l'inventaire des préjudices subis par la victime et, par conséquent, de contrôler « au mieux » le respect du principe de réparation intégrale du préjudice.

\section{II.B.2 L'édiction d'une nouvelle nomenclature}

\footnotetext{
${ }^{96}$ Gardner (D.), «Les victimes d'accidents collectifs : une comparaison franco-québécoise à partir de deux tragédies ferroviaires », Gaz. Pal., 2014, n81, p.
}

1. $\quad{ }^{97}$ Sur la sémantique V. Saison-Demars (J.), « Les « troubles dans les conditions d'existence » dans le droit de la réparation des accidents médicaux », RDSS, 2008, p 890

${ }^{98}$ Gout (O.), « Nomenclature et référentiel », Droit social, 2017, p.944. 
Une dernière solution, rompant également avec l'esprit de la nomenclature, consiste en l'édiction d'une nouvelle nomenclature purgée des déficiences actuelles de la nomenclature Dintilhac. Cette solution semblait être celle portée par le projet de réforme de la responsabilité civile.

En effet, le projet proposé par le garde des Sceaux vise, entre autres, à créer une nomenclature de détermination des préjudices corporels, qui sera formulée par décret, donc par voie réglementaire ${ }^{99}$. Sur ce point, le projet de réforme vise l'égalité de traitement pour toutes les victimes d'un dommage corporel, sur une meilleure prévisibilité juridique et sur une rationalisation encore plus forte de l'évaluation du dommage.

Cette volonté d'une nomenclature règlementaire n'est pas nouvelle et fait notamment écho à certaines sources : la loi Lefrand ${ }^{100}$, la proposition de loi dite Béteille ${ }^{101}$, la loi Fourcade ${ }^{102}$, le projet Terré $^{103}$, certaines références doctrinales ${ }^{104}$, et plus récemment le projet avorté de décret Taubira de décembre $2014^{105}$.

Toutefois, le décret de décembre 2014 précité prévoyait de mettre en partie fin, à la dysmétrie de la nomenclature Dintilhac, en autonomisant les souffrances endurées après consolidation ou la tierce personne des frais divers ${ }^{106}$ alors que dans le projet de réforme actuel rien ne laisse suggérer une

2. $\quad{ }^{99}$ Le projet est disponible à partir de l'adresse du site internet de la chancellerie : http://www.justice.gouv.fr/publication/Projet_de_reforme_de_la_responsabilite_civile_13032017.pdf. Sur le sujet V. Groupe de travail de l'institut DroitSanté, « Projet de réforme de la responsabilité civile et santé », RDSS, 2016, p. 904 ; Borghetti (J.S.), «Un pas de plus vers la réforme de la responsabilité civile : présentation du projet de réforme rendu public le 13 mars 2017 », D., 2017, p. 770 ; Mekki (M.), « Le projet de réforme du droit de la responsabilité civile : maintenir, renforcer et enrichir les fonctions de la responsabilité civile », disponible sur : http://www.mekki.fr/files/sites/37/2016/06/redaction-definitive.pdf ; Borghetti (J.S.), «L'avantprojet de réforme de la responsabilité civile », D., 2016, p. 1386

${ }^{100} \mathrm{http}: / / \mathrm{www}$.assemblee-nationale.fr/13/propositions/pion2055.asp

${ }_{101}$ «L'article 1386-27 pose le principe de l'évaluation distincte des chefs de préjudice par le juge et l'obligation faite au juge, en cas de dommage corporel, de se référer à une nomenclature ainsi qu'à un barème national d'invalidité régulièrement mis à jour, définis par voie réglementaire. » V. https://www.senat.fr/leg/ppl09-657.html

${ }^{102}$ Loi $^{\circ} 2011-940$ du 10 août 2011 modifiant certaines dispositions de la loi $\mathrm{n}^{\circ} 2009-879$ du 21 juillet 2009 portant réforme de l'hôpital et relative aux patients, à la santé et aux territoires, ,JORF, nº167 du 22 juillet 2009, p. 12184 texte $\mathrm{n}^{\circ} 1$. L'article 56-II a été jugé comme un cavalier législatif et donc contraire à la constitution : V. Cons. const., 4 août 2011, n²011-640 DC et son commentaire (p.11) disponible sur http://www.conseil-constitutionnel.fr/conseilconstitutionnel/root/bank/download/2011640DCccc_640dc.pdf

${ }^{103}$ En ce sens V. https://www.courdecassation.fr/IMG/reforme-droit-RC.pdf

3. $\quad{ }^{104}$ En autre : Viney (G.), op. cit.

${ }^{105}$ Projet de décret instaurant une nomenclature des postes de préjudices résultant d'un dommage corporel disponible sur : http://www.justice.gouv.fr/publication/dacs/consult/20141120-projetdecret.pdf ; sur ce sujet V. Clerc-Renaud (L.), «Chronique droit médical/dommage corporel », Droit, Santé et Société, 2016, n², p.43.

${ }^{106}$ V. annexes disponible sur : http://www.justice.gouv.fr/publication/dacs/consult/20141120-projetannexe.pdf 
telle ambition. En effet, des propres mots du garde des Sceaux ${ }^{107}$, il ne s'agit ni plus ni moins que de consacrer par voie règlementaire la nomenclature Dintilhac, sans corriger les erreurs de fond et de forme que nous avons pu soulever plus haut.

Par ailleurs, bien que cette nomenclature soit annoncée comme «non limitative », selon l'article $1269 \mathrm{du}$ projet de réforme, son caractère règlementaire empêcherait les magistrats de façonner sur mesure des postes de préjudice pour leurs cas particuliers, puisqu'ils seraient liés par les définitions des postes de préjudices prévus par le décret ${ }^{108}$. Le projet de réforme fait de la nomenclature - dont la normativité «douce » ${ }^{109}$ est d'ores et déjà contraignante, on l'a vu - une norme «dure », figée, restrictive, par laquelle tous les acteurs du dommage corporel seraient liés. L'édiction d'une nomenclature réglementaire pourrait entraîner un risque accru de violation du principe de réparation intégrale du préjudice. Comme on a pu le souligner : «tout classement produit - ou finit par produire - une contrainte cognitive qui peut aboutir, par la force de l'habitude, à négliger des préjudices ne rentrant pas dans le cadre institué ${ }^{110}$. Autrement dit, la standardisation du processus d'indemnisation conduit à des réflexes mécaniques qui dispensent le juge d'individualiser et de réfléchir sur les préjudices qui lui sont soumis ${ }^{111}$ ce qui peut conduire à méconnaitre de nouveaux préjudices. Cette standardisation étant indéniablement corrélée au niveau de la force contraignante $\mathrm{du} \ll$ classement $»$ en question.

Cela étant, on pourrait croire que l'article 1267 du projet, lequel prévoit que les dispositions de cette nomenclature règlementaire s'appliquent «aux décisions des juridictions judiciaires et administratives », est une avancée majeure. Le domaine étendu prévu pour la nomenclature révèle la volonté de la chancellerie de garantir l'égalité de traitement des victimes devant toutes les juridictions, judiciaires comme administratives. C'est une orientation louable au regard des efforts de rapprochement entre les deux juridictions, que l'on observe clairement depuis la promulgation de

\footnotetext{
${ }^{107}$ Kleitz (C.), Urvoas (J.J), « « Le projet de réforme de la responsabilité civile est une œuvre collective portée par la Chancellerie » », Gaz. Pal., 2017, n¹1, p.10.

${ }^{108}$ Derepas (L.), Le Cheualier (E.) et Quézel-Ambrunaz (C.), «Regards croisés sur une innovation. Le recours poste par poste : bilan et perspective », Gaz. Pal., n hors-série 1, p.14.

${ }^{109}$ Bacache (M.), op. cit., p.7 ; sur le droit souple V. Maurin (L.), « le droit souple de la responsabilité civile », RTD civ., 2015 , p. 517.

${ }^{110}$ Prévost (J.B.), «Le préjudice exceptionnel : réflexions sur la fonction et les limites de la nomenclature des postes de préjudice », Gaz. Pal., 2014, n056, p.

111 Pasquier (T.), «Le préjudice à la croisée des chemins », Rev. Trav., 2015, p. 741 ; Arcadio (D.), op. cit : cette réflexion porte initialement sur les barèmes et référentiels d'indemnisation du dommage corporel mais semble ici tout à fait pertinente et extrapolable à notre espèce.
} 
la loi $\mathrm{n}^{\circ}$ 2002-303 du 4 mars 2002 ${ }^{112}$. Cependant, cette «avancée majeure » est à relativiser, d'une part, puisque le droit administratif et le droit civil s'étaient déjà - certes péniblement - accordés sur l'emploi de la nomenclature Dintilhac, et d'autre part car les régleurs des procédures d'indemnisation amiables et les CCI ne sont pas inclus dans ce dispositif. Si cette nouvelle nomenclature s'avère différente de la nomenclature Dintilhac et si ces organismes décident de ne pas la suivre, cela pourrait occasionner des disparités d'indemnisation selon le type de procédure ${ }^{113}$, ce qui est à contre-courant de l'esprit de la réforme.

Finalement, on mesure que le souhait de la chancellerie de renforcer l'égalité de traitement devant toutes les juridictions, la meilleure prévisibilité juridique, et la rationalisation encore plus forte de l'évaluation du dommage corporel, constituent un moindre bénéfice au regard des risques engendrés par l'adoption d'une nomenclature par voie réglementaire, qui n'ambitionne pas de corriger les défectuosités actuelles de la nomenclature Dintilhac.

Pour conclure, gardons à l'esprit que la nomenclature ne doit être qu'une «boussole » et non un «instrument managérial » ${ }^{114}$.

\section{Conflits d'intérêts : aucun}

\footnotetext{
${ }^{112}$ En ce sens Bacache (M.), Laude (A.), Tabuteau (D.), « La loi du 4 mars 2002 relative aux droits des malades, 10 ans après », Paris, Bruylant, « collection du centre de droit médical et biomédical », 2013, 334 p.

${ }^{113}$ Ce qui est déjà le cas. En ce sens Bouvet (S.), « L'équité dans la réparation du préjudice : de quelle équité me parlezvous ? », RCA, 2007, n5; Bouvet (S.), «De quelle équité me parlez-vous ? (où l'égalité prend le pas sur l'équité...) », disponible sur : https://www.courdecassation.fr/venements_23/colloques_4/2006_55/me_parlez_9658.html; «L'indemnisation du dommage corporel: de la disparité à l'harmonisation», Colloque de l'université de Picardie Jules Verne, RGDM, 2009, no 31 ; Morlet-Haïdara (L.) «Un droit en miette ou le mille-feuille de la réparation du dommage corporel», RGDM, 2009, no 31, p. 27 et s.; Livre blanc sur le dommage corporel, FFSA 2008, p. 8 et 9, disponible sur : http://www.gazetteassurance.fr/wp-content/uploads/2015/02/Livre-blanc-FFSA-GEMA-sur-lindemnisation-dudommage-corporel-avril-2008.pdf; Porchy-Simon (S.), Gout (O.), Soustelle (P.), Augier (E.), Bascoulergue (A.), De Jong (N.), Granier (C.), Ménard (B.), Rias (N.), Waltz-Teracol (B.), et al., «Étude comparative des indemnisations des dommages corporels devant les juridictions judiciaires et administratives en matière d'accidents médicaux », Rapport de Recherche, Mission de recherche Droit et Justice. 2016, pp.324 : la conclusion est la suivante «le niveau d'indemnisation par le juge administratif apparait globalement inférieur, voire très inférieur, à celui du juge judiciaire ».

Disponible sur : https://halshs.archives-ouvertes.fr/halshs-01360837/document

${ }^{114}$ Prévost (J.B), «L'homme moyen ou l'étalon vide... », Gaz. Pal., 2012, n³15, p. 7.
} 